\section{Trauma Surgery} \& Acute Care Open

\title{
Refractory pelvic fracture-associated hemorrhage after preperitoneal packing: now what?
}

\author{
Gretchen Floan, 1,2 Matthew J Martin (1) 1,2
}

'Department of Trauma Surgery, Scripps Mercy Hospital San Diego, San Diego, California, USA

${ }^{2}$ Department of Surgery, Naval Medical Center San Diego, San Diego, California, USA

Correspondence to Dr Matthew J Martin; traumadoc22@gmail.com
(C) Author(s) (or their employer(s)) 2020. Re-use permitted under CC BY-NC. No commercial re-use. See rights and permissions. Published by BMJ.

To cite: Floan G, Martin MJ. Trauma Surg Acute Care Open 2020;5:e000629.

\section{CASE DESCRIPTION}

A male patient in his 50 s presented after a highspeed motorcycle crash. On arrival to the trauma bay, his primary survey was intact with normal vital signs. He had an obvious deformity of his left forearm secondary to a closed radius/ulnar fracture. He had anterior pelvic tenderness but no perineal/ scrotal bruising. A pelvic X-ray and CT scan of his torso showed severe diastasis of the pubic symphysis with associated posterior disruption of the sacroiliac joint (figure 1A). He had a small associated extraperitoneal pelvic hematoma extending into the left rectus sheath with trace arterial blush and mild hemorrhage into the urinary bladder. He remained hemodynamically stable with a Glasgow Coma Scale score of 15 and with the main complaint of pain from the left forearm fracture.

The patient was taken immediately to the operating room (OR) for planned external pelvic

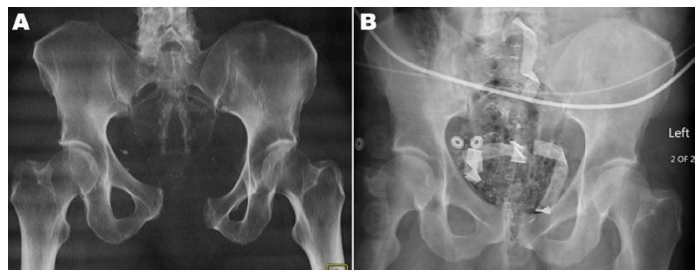

Figure 1 (A) Initial pelvic X-ray showing severe pubic symphysis diastasis and posterior disruption of the left sacroiliac joint, and (B) immediate postoperative X-ray showing the QuikClot Control+ pads in the preperitoneal space and adequate pelvic reduction obtained via placement of an external pelvic binder.

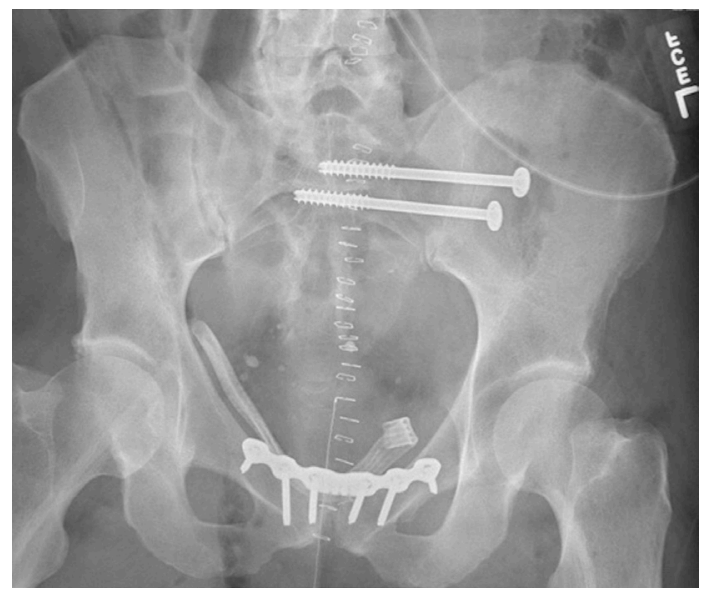

Figure 3 Postoperative X-ray demonstrating operative pelvic fixation with anterior pubic symphyseal plating and left sacroiliac screw placement.

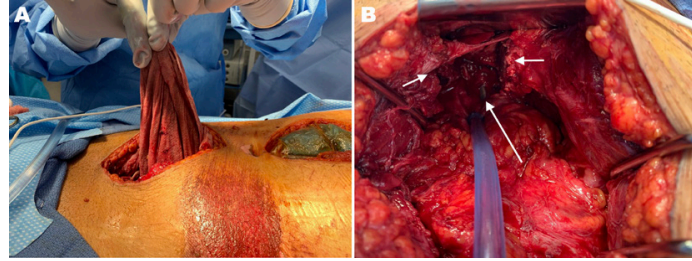

Figure 2 (A) Intraoperative photo showing the upper midline laparotomy incision and the lower midline incision used for preperitoneal pelvic packing, with QuikClot Control+ lap sponges being removed. (B) Large preperitoneal pelvic cavity with complete hemostasis after packing removal (at 36 hours). Short arrows mark the left and right pubic symphyseal edges demonstrating severe diastasis and long arrow demonstrating the anterior urethral defect with exposed Foley catheter.

fixation and fixation of his left forearm. On anesthetic induction, he became acutely hypotensive and went into pulseless electrical activity cardiac arrest. $\mathrm{He}$ required a brief period of cardiopulmonary resuscitation and responded to $1 \mathrm{mg}$ epinephrine with return of spontaneous circulation. Massive transfusion protocol was initiated with cold-stored low-titer type $\mathrm{O}$ whole blood. A rapid mini upper abdominal laparotomy was performed without signs of intraperitoneal hemorrhage, and a simultaneous infraumbilical midline incision was made to perform pelvic exploration and preperitoneal packing. A significant amount of clot and blood was evacuated, exposing a large cavity within the pelvis with active arterial and venous hemorrhage. Several branches of both internal iliac arteries and veins were ligated. A $3 \mathrm{~cm}$ defect in the anterior urethra was detected at its junction with the bladder but was difficult to fully visualize due to active bleeding. Despite packing with six laparotomy pads, there was ongoing bleeding from multiple areas that soaked through the pads. The patient remained hemodynamically unstable and hypotensive.

\section{WHAT WOULD YOU DO NEXT?}

A. Place a resuscitative endovascular balloon occlusion of the aorta catheter.

B. Dissect further into the pelvis to identify the source(s) of bleeding.

C. Identify and ligate or clip bilateral internal iliac arteries.

D. Transport the patient to the interventional $\mathrm{ra}-$ diology (IR) suite or hybrid OR for pelvic angioembolization.

E. Remove and replace the laparotomy pads with advanced hemostatic sponges/dressings. 


\section{WHAT WE DID AND WHY}

The six standard laparotomy pads were removed and replaced with four large (12 x 12 inch) QuikClot Control+ $₫$ (Z-Medica, Wallingford, Connecticut) hemostatic gauze lap sponges into the preperitoneal space. This resulted in immediate hemostasis and with no blood soaking through or leaking around the packing. The patient rapidly stabilized and the transfusion of blood products was halted. A complete abdominal exploration via the upper midline incision was then performed, which allowed a 10-15 minute period to observe for any additional bleeding from the pelvis. No intra-abdominal injuries were discovered and there was no additional pelvic hemorrhage. The anterior rectus sheath fascia was temporarily reapproximated over the preperitoneal packing and temporary closure of both incisions was performed using negative pressure dressings and sponges. An external pelvic binder was then applied with adequate reduction of the pelvic diastasis (figure 1B). The patient was transferred to the intensive care unit for further resuscitation and close monitoring. He required no additional blood product transfusion during the next 2 days.

He was taken back to the OR 36 hours later where the pelvic packs were removed and one additional bleeding vessel was suture ligated. Otherwise, the large preperitoneal cavity was now entirely hemostatic (figure 2). The urethral injury found during the initial operation could now be adequately exposed, visualized, and primarily repaired over the Foley catheter. Orthopedic surgery also repaired the pelvis at this time with anterior plating of the pubic symphysis and sacral screws (figure 3). A Jackson-Pratt drain was left in the pelvic cavity adjacent to the urethral repair, and formal closure of both the laparotomy and the pelvic packing incisions was performed in standard fashion. The patient was extubated the following day, had gradual return of bowel function, and recovery from a mild acute kidney injury. He was transferred to the trauma ward on hospital day 7 where he continued to progress well and had an uncomplicated postoperative recovery. He was discharged to home on hospital day 18.
Multiple studies in accordance with recent combat experience have found advanced hemostatic dressings to be superior to standard gauze products for achieving hemostasis in a variety of injury types and patterns, and in the setting of traumatic coagulopathy. However, until recently there were no hemostatic gauze products designed and approved specifically for internal use as in this case. The product used in this case is a more recently developed kaolin-coated sponge similar to standard laparotomy pads that is specifically designed and approved for internal use. In this situation, it was the easiest and fastest maneuver that allowed us to stabilize the patient and achieve immediate control of bleeding. This maneuver also spared further dissection into the pelvis (with likely additional large blood loss) or transportation of this unstable patient to another location such as the IR suite for angioembolization. If repacking with these hemostatic pads failed, our most likely next step would have been clip ligation of the bilateral internal iliac arteries which is not a decision without consequences.

Contributors Both coauthors have contributed to the data collection, article drafting, revision, and final approval.

Funding The authors have not declared a specific grant for this research from any funding agency in the public, commercial or not-for-profit sectors.

Disclaimer The viewpoints and opinions expressed in this case report are those of the individual authors and do not reflect those of Scripps Mercy Hospital, the Naval Medical Center San Diego, or the Department of Defense.

Competing interests None declared.

Patient consent for publication Obtained.

Provenance and peer review Not commissioned; internally peer reviewed.

Open access This is an open access article distributed in accordance with the Creative Commons Attribution Non Commercial (CC BY-NC 4.0) license, which permits others to distribute, remix, adapt, build upon this work non-commercially, and license their derivative works on different terms, provided the original work is properly cited, appropriate credit is given, any changes made indicated, and the use is non-commercial. See: http://creativecommons.org/licenses/by-nc/4.0/.

ORCID iD

Matthew J Martin http://orcid.org/0000-0002-9169-9069 